urethrostomy will, it is held, be found to yield the best results. Post-operatively it confers an increased margin of safety on complete closure after operations on the bladder or prostate. Furthermore drainage can be maintained indefinitely in order to ensure sound healing of the abdominal wound.

\section{BIBLIOGRAPHY}

BARNEY, J. D. (1934), Trans. Amer. Ass. Gen.-Urin. Surg., 27, 73. DEMING, C. L. (1947), four. Urol., 57, 49.

DUKES, C. E. (1929), Proc. $R$. Soc. Med., 22, I.

FREYER, P. J. (1908), 'Clinical Lectures on Surgical Diseases of the U' Urinary Organs,' London.
FULLERTON, A. (1913), Brit. Med. Four., 1, 332.

GALBRAITH, W. (1948), Proc. $R$. Soc. Med., 4r, 73.

HARRISON, R. (1878), 'Clinical Lectures on Stricture of the Urethra and other Disorders of the Urinary Organs,' London.

HEY, W. H. (1945), Brit. Med. Four., 2, 91 .

LEWIS, L. G. (1943), Bull. U.S. Army Med. Dept., 69, 46.

LYNN-THOMAS, J. (1914), Lancet, I, 1456.

PRATHER, G. C. (1947), four. Urol., 57, 15

RATHBUN, N. P. (1934), Trans. Amer. Ass. Gen.-Urin. Surg., $27,47$.

REES, SKYRME W. (1947), Brit. Four. Urol., 18, 83.

RICHES, E. W. (1943), Brit. Four. Surg.. 31, 135.

THOMPSON, H. (1879), 'Clinical Lectures on Diseases of the Urinary Organs,' London.

YOUNG, H. H. (1926), 'Practice of Urol,' Philadelphia, Vol. 2, 600.

YOUNG, H. H. (1934), Trans. Amer. Ass. Gen.-Urin. Surg., 27, 7 I.

\title{
THE ISCHAEMIC LIMB
}

\author{
By Peter Martin, M.Chir. \\ From the Department of Vascular Surgery, Post Gradiate Medical Sch)ol, London; Surgeon, Chelmsford and \\ Essex Hospital
}

The proper nutrition of a limb depends on, amongst- other things, a sufficient blood supply. The main arteries to a limb may become obstructed to a remarkable degree, in fact, they may be completely obstructed without the patient's knowledge, and often without his medical attendant's knowledge, because alternative pathways exist. Apart from sufficient patency of the vessels, an adequate cardiac output is necessary, which depends on sufficient cardiac return and consequently a proper blood volume. Thus in severe arterial trauma causing an interruption of a main vessel, the life of the limb may become precarious, not only as a result of damaged vessels but also of a diminished blood volume resulting from severe haemorrhage at the time of the incident, and a timely and possibly massive blood transfusion may well tilt the scale towards recovery of the limb. Thus the factor of anaemia must be considered as well as the patency of the vessels.

Ischaemia of a limb may occur in the following conditions :

(1) Trauma, causing rupture, contusion and thrombosis, spasm or later aneurysm of an artery.

(2) Embolism, from a fibrillating auricle or valvular vegetation, from atheromatous plaques in the largest vessels, and in paradoxical embolism, where there is patency between the right and left side of the heart, from the venous system.

(3) Thrombosis, where the vessel wall is diseased as in arterio-sclerosis, thromboangiitis obliterans or syphilis, or when the vessel is compressed, as in cervical rib, or invaded or compressed by tumour, or in severe blood diseases and infective conditions such as typhoid or pneumonia, the seat of intraluminar clot.

(4) The so-called spontaneous monarteritis of indeterminate origin (Learmonth), in which there is no overt cause of thrombosis.

(5) Certain spasmodic conditions such as occur in the Raynaud syndrome, traumatic arterial spasm and, it may be, as a reflex phenomenon in certain cases of venous thrombosis, though this must be rare.

Apart from trauma and spasm it will be seen that arterial obstruction results from embolism or thrombosis, and even in these two conditions thrombosis is generally the factor which finally seals the fate of the limb.

\section{Morbid Anatomy}

Whether the original obstruction be due to thrombosis or embolism the important factor is the rapid addition of clot with blockage of valuable collateral vessels, further embarrassing the circulation. A clot may extend widely, even through the whole length of a limb, and from the distal end of this clot fragments may break off and block more distal vessels previously unaffected. This is not infrequently seen when a rib presses on the subclavian artery (resulting in dilatation beyond the point of pressure, together with the formation 
in the dilated part of clot which may fragment and cause embolic phenomena in the fingers.

Ten to twenty-four hours after an embolus has lodged or thrombosis has occurred, an intimal reaction occurs, the clot becomes adherent and the vessel becomes swollen, tender and painful. In embolism the original source of the embolus must not be overlooked. Fibrillation or flutter may have recently given place to a normal rhythm, or may still be present ; the importance of this will be further considered.

The effects of a complete obstruction to the circulation of a limb are identical with those which follow the immediate application of a tourniquet.

Temferature changes. The limb gradually loses its heat and assumes the temperature of its surroundings, the distal parts cooling more rapidly than the more proximal owing to their relatively larger surface area. The limb does not become icy cold; in fact it can only reach a temperature below its surrounding atmosphere if evaporation takes place, and then only of a degree or two. This is a gradual process and not a sudden phenomenon as so often described. An ischaemic limb warmed by blankets may have a temperature nearly as high as its contralateral, normal fellow. Before examination for temperature changes, both limbs should be exposed to room temperature for at least half-an-hour when any difference becomes apparent and significant.'

Colour changes. At first the limb becomes pale and white, but in a few minutes a blue cyanotic tinge appears due to reflux of venous blood into the capillaries. This cyanosis gradually deepens from a capillary dilatation due to the direct action of local metabolites and products of anoxia, on the vessel walls. The colour also deepens in warm surroundings and, alternatively, if the part is cooled to a temperature of $15^{\circ} \mathrm{C}$, the colour is red owing to the fact that oxyhaemoglobin does not dissociate readily at and below this temperature. After three to five minutes white spots (Biers spots) appear, due to a local spasm of arterioles. The origin of this spasm is unknown and if there is a free venous outlet the spots increase in size, gradually coalescing, and the limb assumes a uniform pallor.

\section{Effects on the Structures of a Limb}

On nerves the effect is a paralysis, centripetal in progress and becoming complete in 45-60 minutes. When the blood flow is resumed recovery occurs, but irreversible changes occur in the nerves if the ischaemia is maintained complete for 12 hours or more.

The muscles as a result of the failure in function of the nerves become paralysed after about $45 \stackrel{0}{0}$ minutes, although they are excitable to the 3 faradic current long after this time-in fact, until ${ }^{\Phi}$ infarction and necrosis supervene, and these. irreversible changes occur sooner than in nerve $\vec{F}$ tissue, after about 6-8 hours. The result of a blockage of blood flow to a muscle is not apparent? to the patient unless the muscle contracts. Then $\frac{\overline{\bar{D}}}{\bar{\rho}}$ severe pain of a cramping nature occurs and $\frac{\vec{\sigma}}{\vec{\sigma}}$ remains until some blood flow is resumed and the $\triangle$ local $\mathrm{m}$ tabolites, the cause of the pain, are washed $\%$ away or, alternatively, until continued ischaemia $\vec{\circ}$ results in paralysis of the pain-conducting fibres of the nerves. Infarction and necrosis of muscle $\vec{\omega}$ is followed by fibrosis and contracture-e.g. Volkmann's ischaemic contracture. A lesser? degree of ischaemia of muscle is evidenced clinically by intermittent claudication.

It will be seen from the above description of the results of complete vascular obstruction, that the symptoms are not of sudden but of gradual onset, $\checkmark$ the rapidity of their appearance depending upon the activity of the muscles of the limb at the time of the incident. A limb which is in active use will 7 very soon give rise to muscle pain, but a limb 을 which is lying in bed may suffer complete blockage to a main vessel without any symptoms, if the collateral circulation is adequate, only to $\overrightarrow{0}$ diagnosed at a later date by the incidental dis covery of absent distal pulses.

Embolism at the aortic bifurcation becomes apparent to the patient when he discovers that his legs are paralysed; subsequently it is noticed that they are cold, sometimes only after they have $\frac{\mathbb{Q}}{\mathbb{Q}}$ been exposed to room temperature for some time $\stackrel{2}{\vec{D}}$ for a somewhat prolonged clinical examination. $\frac{0}{3}$ It may be, however, in aortic embolism that the paralysis is of more sudden onset ; obstruction of the blood flow in the aorta may cause an ischaemia of the lower segments of the cord, as a result of 3 which not only voluntary muscles but also the sphincters may be involved.

The above description is usually considerably $\delta$ modified by the fact that ischaemia is rarely complete. Establishment of the collateral circulation $ᄋ$ nearly always occurs to some extent and may occur rapidly.

\section{The Collateral Girculation}

This is a remarkably efficient system-in fact, $N$ in a young person with a healthy vascular tree the $N_{\omega}$ main vessel to a limb can be ligatured at any level with little apparent effect on the function of the 0 limb. When the vessels are diseased the collaterals are also involved and compensation is $\stackrel{\oplus}{+}$ often inadequate. Immediately after obstruction $T$ to the main stem the collateral vessels dilate, not $\stackrel{0}{\circ}$ as the result of a supposedly increased vis-a-tergo 
which it can be shown does not occur, but rather as a result of a diminished resistance due to emptiness of the distal vessels and to local dilatation of these, vessels by the presence of tissue metabolites resulting from the anoxaemia. This process occurs in the course of hours or days. An actual growth in length and girth of the collaterals also takes place but is very much more protracted, taking months or even years. It is considerable in 10 to 12 weeks. The whole process may be seriously modified if thrombosis spreads from the site of obstruction blocking the mouths of collaterals and even spreading into the collaterals themselves. It appears that the collateral circulation occurs largely in the subcutaneous tissues and fascial planes rather than in the muscles, and this accounts for the high skin temperatures often seen in a limb with absent peripheral pulses and even in a limb with gangrene of the digits. In cases of severe arterial trauma, the surrounding tissues may be so damaged immediately or remotely by bruising and subsequent thrombosis that the potential collateral circulation is not available. Under these circumstances the condition of the limb is indeed serious.

Clinically the signs and symptoms of ischaemia of a limb may be grouped as follows:

(I) Effects on colour.

(2) Effect on skin and subcutaneous tissues.

(3) Effects on deep - structures, especially muscles.

(4) Effects on temperature and pulses.

(5) Effects on nerves.

(i) The colour changes in an ischaemic limb are very significant. The normal hand or foot, on elevation, slowly assumes a pallor which disappears within 15 to 30 seconds after return to the heart level. This experiment is easily performed, and offers a simple test for normal limb circulation. In chronic arterial disease the pallor becomes more intense within a shorter time and there may be a delay of 60 seconds or more before colour returns on resumption of the heart level. In occlusive vascular disease the pallor may become apparent at an angle less than $90^{\circ}$ (Critical angle). If blood is flowing slowly through the skin, it has more time to give up its oxygen and therefore the skin assumes a variable degree of cyanosis. A violaceous tint indicates almost complete stoppage of the circulation. Should the part be cold there will be little dissociation of oxyhaemoglobin, the blood will remain oxygenated and the skin be a brick-red colour (Rubor dolorosa). Conversely if an ischaemic limb is warmed cyanosis will develop more rapidly and more intensely. A slight degree of cyanosis of the hand or foot is a common and normal physiological reaction at temperatures between 14 and 25 degrees centrigrade.

Sir Thomas Lewis summarized the colour changes in skin as follows :-

Warm pale skin-warm, because of rapid blood flow ; pink, because there is plenty of oxygen and pale because the skin is well nourished and therefore the minute vessel tone is high.

Warm deeply coloured red skin-such skin has been irritated by heat or some other agency and is in a state of inflammation, or else cutaneous vasodilation has been brought about through nervous channels or by means of drugs-e.g. Amyl nitrite.

Warm cyanosed skin-the supply of blood is imperfect; the limb has been warmed artificially - e.g. by lying under the bed clothes.

Cold pale cyanosed skin-the blood flow is very slow or absent. The deeper the tint, the more defective is the circulation.

Cold deeply coloured red skin-below $10^{\circ} \mathrm{C}$, oxyhaemoglobin is not dissociated but the minute vessels are $d$ : maged and dilate.

The rapidity of return of colour in the skin after digital pressure is not necessarily of value as a test of circulatory efficiency, for a rapid return of colour may result from reflux into the capillaries of a limb in which the circulation is completely stationary. Thus as a test for tightness or otherwise of a plaster cast the test is useless.

(2) The effects of ischaemia on skin and subcutaneous tissues are seen in atrophy of these structures. The skin loses its corrugations and becomes shiny and inelastic, appears somewhat papery and loses its hairs. As a result of atrophy of subcutaneous tissues the skin cannot be so readily picked up between the fingers. There is ridging and irregularity of the nails and a tendency to persistent ulcers, cracks and fissures and to recurrent whitlows, especially paronychiae. Eventually patches of superficial gangrene may occur. There is also a persistent coldness of the affected part.

(3) The effect of ischaemia of deep structures is seen largely in muscles. Three grades of reaction may be encountered. (i) First, fibrillation of muscle is an early sign of an inadequate blood supply and is often detected readily in the abductor hallucis in the lower limb. It may have to be observed for a minute or two. (I I) A more serious result of ischaemia is intermittent claudication which may, of course, occur in any muscle group, and is not confined to the calf muscles. It is not uncommon for claudication in the muscles of the foot to be treated as flat foot or foot strain. (iii) Finally muscle infarction and contracture as in Volkmann's ischaemia contracture result when the muscle fibres die from lack of oxygen and are replaced by a tough unyielding fibrous tissue. 
(4) Pulses may be diminished or absent throughnut the limb, but certain factors must be taken into consideration in testing these. The limb to be tested should be comfortably warmed and the pulses on each side should be compared. Abnormalities in anatomy should be remembered before absence of a pulse is assumed-e.g. the peroneal artery may take the place of the Dorsalis Pedis and the radial may pass to the back of the hand above the wrist joint. It is the difference in one limb compared with its fellow that is important, both as regards temperature, colour and pulse, rather than the observation of these phenomena in a single limb. The oscillometer is a useful instrument for detecting pulsation, especially in the presence of oedema when the pulses may be difficult to feel.

(5) The effect on nerves is paralysis provided the ischaemia is complete or almost so. In somewhat less severe cases pain, occasionatly confined to the distribution of a particular nerve but more usually in an irregular distribution, may occur when the limb is at rest, particularly at night. It may be very severe, constant or paroxysmal, and occasionally of a severe burning character. In cases of thromboangiitis obliterans it is often exceedingly severe but is generally not so marked in cases of arteriosclerosis. It may be that this pain is due to an ischaemic neuritis and Priestly has demonstrated microscopical changes in the nerve trunks in these cases. Section of nerve trunks to relieve the pain is rarely successful and it is not entirely proven that the pain of an ischaemic limb is in fact due to ischaemic neuritis.

The Raynaud phenomenon is a common symptom in deficiency of the circulation and may often be the first sign of obstructive vascular disease.

\section{Treatment}

This is considered under four headings.

(I) The prevention of increasing embarrassment of the circulation.

(2) The encouragement of a collateral circulation.

(3) The preservation of the tissues of the limb until the blood flow is, if possible, re-established.

(4) Operative measures.

(I) The differential diagnosis between thrombosis and embolism of a main artery is impossible apart from the presence of some predisposing factor. If the heart is fibrillating then there is good reason to suspect embolism ; in advanced arteriosclerosis, thrombosis would be expected. However the obstruction is caused, it is most important in acute cases, especially embolic cases, to prevent the addition of further clot and blocking of the mouths of collateral vessels. Anticoagulants are given at the very earliest moment even before the patient is transferred to hospital. Fifteen thousand units of heparin are injected intravenously. Treatment with dicoumarol is started as soon as the patient is in hospital and 300 mgms. are given by mouth. The heparin is repeated in doses of 10,000 units 6-hourly and the prothrombin index is estimated as soon as possible. The prothrombin index is of more value than the prothrombin time, the latter is variable and unreliable, whereas the former is specific for any particular patient. It is not necessary to estimate the bleeding time with the administration of heparin, as in the doses mentioned haemorrhages will not occur. On the second day a further dose of $200 \mathrm{mgm}$. of dicoumarol is given and this is repeated daily, the object being to achieve a prothrombin index of 20 to 40 per cent. Should the index fall below 20 per cent. the dose of dicoumarol is reduced to roo mgm. or even omitted. A patient on heparin and dicoumarol has the heparin stopped when the prothrombin index has reached a figure of 20 to 40 per cent., but the dicoumarol is continued for at least ro days after the vascular incident: It is essential for a daily prothrombin index to be obtained. Haemorrhage from over-dosage of heparin requires the administration of protamine sulphate in a dose of 5 to ro cubic centimetres of a I per cent. solution intravenously. Overs dosage of dicoumarol is treated by Vitamin Ko음 $4 \mathrm{mgm}$. of the synthetic menadione intravenously together with a fresh blood transfusion. When embolism is associated with a fibrillating heart it is better not to try to bring the heart into control unless heart failure is imminent. The return of a normal heart rhythm may well be associated with further embolic phenomena.

There appears to be considerable divergence of opinion as to whether, after the occurrence of an embolism or thrombosis, there is spasm of the collateral vessels. I believe that the evidence of active spasm is not very substantial but it is true that some hours elapse before the collaterals open fully. These vessels dilate in accordance with the mechanism previously described. It is doubtful if at this stage a sympathectomy is either indicated or warranted. The patient is often in poor shape to stand such an operation and sympathectomy by injection of alcohol or 1o per cent. phenol as suggested by Boyd is difficult and uncertain except in expert hands. Deep sleep is always accompanied by complete sympathetic release and this can be secured with the help of such barbiturates as sodium amytal. At the same time the body and unaffected limb are kept warm under bedclothes to induce reflex vaso-dilation. Alcohol, the best vascular dilator we have, is given in liberal doses and a very adequate sympathetic release is achieved 
in a way that is acceptable to the patient. At a later date a preganglionic sympathectomy may be of distinct value if reflex heating and spinal analgesia reveal vessels which are still capable of dilating. It is felt that sympathectomy by operation has no place in the early stages of acute vascular lesions. Later, and in chronic arterial disease, it is sometimes of value in dilating subcutaneous channels and thereby increasing the collateral bed available to bye-pass the blood round the blocked main vessel. I would estimate that sympathectomy is indicated by release tests in some 50 per cent. of arteriosclerotics with threatened gangrene under the age of 60 and in some cases above this age. Employment of sympathectomy in this way excludes the problematical risk of shumting blood from the more intolerant muscles to the skin and subcutaneous tissues which can stand ischaemia better. Intermittent venous occlusion, intermittent pressure and suction, the oscillating bed, postural exercises, intravenous hypertonic saline, the injection of tissue extracts and the exhibition of a varied collection of drugs, vitamins and hormones seem to be of little or no value in improving local circulation.

(3) The ischaemic limb is kept scrupulously clean and handled with the utmost care and gentleness. If gangrene is threatened or established the limb is afforded complete rest in the horizontal position and it is not elevated. Dependency is equally to be deprecated as it leads to venous embarrassment. It is of the utmost importance that the limb be kept cool at a temperature of about $20^{\circ} \mathrm{C}$; at this temperature the local metabolism, and consequently the tissue demands for oxygen, are reduced to a minimum. Above this temperature, the local metabolic rate increases very rapidly indeed and with it the demands for blood. If blood flow is not available for the tissues. irreparable damage is readily done by the accumulation of the waste products of metabolism. Icing has been recommended, but may damage the tissues unduly ; moreover at temperatures below $15^{\circ} \mathrm{C}$, dissociation of oxyhaemoglobin practically ceases and the circulating blood is of little value. A temperature of $20^{\circ} \mathrm{C}$ is usually attained reasonably closely by leaving the limb uncovered outside the bedclothes. Locally, the treatment of gangrene includes the application of moist dressings of Eusol and the removal of the constricting sloughs, crusts and exudates which may cover up and constrict viable tissues, and warm and weak antiseptic baths twice a day for half an hour or so are useful adjuncts. Infection is combated by chemotherapy, either locally or systemically and any strong antiseptic is of course avoided.

(4) Operative treatment. In aortic embolism the only hope lies in embolectomy, which is done as early as possible. Success can be expected if this is performed within Io to 12 hours, but after this time, although some successes have been recorded, recovery is unlikely and will almost certainly be incomplete. As for emboli in other situations, it is doubtful whether operation is wise. With anticoagulant therapy, proper management of the ischaemic limb and functional sympathetic release secured by sleep, reflex heating and alcohol, the results of conservative treatment are probably superior to those of operative treatment. This is especially so where the arterial tree is reasonably healthy, as it often is when embolism rather than thrombosis is the essential pathology. When operative measures are indicated heparin must be cautiously used, and probably should not be started until a few hours after the operation is completed. Even then it is probably not necessary since the blood flow is fast enough to discourage thrombosis. It is maintained by some that although the clotting time is increased the bleeding time is little affected as there is no interference with the retraction of vessels.

The role of sympathectomy has been discussed and it is stressed that a preganglionic section is the only suitable operation. Periarterial stripping is never indicated.

Recently a surgical attack on the problem of intermittent claudication of the calf muscles hase been carried out with considerable success in? selected cases (Boyd and Learmonth). Through a posterior median incision in the popliteal space the nerves supplying the two heads of the gastrocnemius and the superficial part of the soleus are resected. It is important not to divide the medial sural cutaneous nerve which may arise from the posterior tibial nerve in conjunction with the medial of the two nerves. It is surprising that very little disability follows this operation; the deep part of the soleus and the posterior tibial group of muscles, together with gravity, all contribute to plantar flexion.

A case recently operated on by me was that of a Thames lighterman aged 62 complaining of intermittent claudication with a claudication distance of roo yards, but with healthy skin, subcutaneous tissues and with a palpable femoral pulse. Before operation he had been off work for two months, but he was able to return to his lighters a month after operation. Although he managed well, he had lost some confidence in jumping from one lighter to another, and has had to take employment in a tug. He can now, nine months after operation, walk at least two miles in complete comfort.

Patients for this operation must be chosen with considerable care. Where there is associated evidence of ischaemia of the skin or superficial 
tissues and where the symptoms occur in a case of progressive arterial disease the results are likely to be disappointing, if not definitely harmful. For example, a labourer, aged 64 , presented with claudication in the calf after walking a distance of 60 yards. He had a sympathectomy performed in 1945 with no improvement. The femoral pulse was not palpable on the affected side and there was some loss of hair and atrophy of skin of the affected leg. A popliteal neurectomy was performed in February, 1948 but six weeks later, though there was no claudication in the gastrocnemius there was claudication in the muscles of the feet and in the anterior tibial group and he was also suffering from rest pain The leg is to be amputated for severe ischaemia and pain.

Operation should be limited to patients with an episodic segmental type of vascular obstruction, generally under 60 years of age and with pain limited to a more or less localized part of the gastrocnemius, usually the inner head. If the operation is likely to be successful, it should be possible to abolish the pain of claudication before operation by injection of novocaine into the tender and painful part of the muscle. The superficial structures must be healthy. Indiscriminate popliteal neurectomy will rapidly bring a useful operation into disrepute.

Sympathectomy is never indicated for claudication alone but may often with advantage be combined with a popliteal neurectomy where there is an element of spasm in the vessels of the limb.

Section of nerves for pain is disappointing and rarely of value and ligation of veins is irrational and useless.

Local amputation for gangrene occurring in the middle three toes is often satisfactory, but gangrene occurring in the great or little toes as a general rule requires amputation of the leg. If the popliteal artery is pulsating well there is a reasonable prospect of success with local amputation, but if pulsation of this vessel is imperceptible it is probable that amputation through the leg will shortly become necessary. If gangrene spreads from the toes to the dorsum of the foot, a major amputation is indicated. As regards gangrene of the distal half of the great or little toes, provided the skin does not show excessive atrophy, and with a palpable popliteal artery and especially if there is a line of demarcation, local amputation should be tried with a good chance of success.

There is a choice of three sites for major amputation-a 5 inch tibial stump, a 10 inch to 12 inch femoral stump and a supracondylar amputation of the Stokes-Gritti type. Amputation through the tibia should be successful where the popliteal artery is pulsating, but where this vessel is blocked amputation should undoubtedly be through the femur. The femoral stump, whether it be 10 inch to 12 inch or supracondylar, always heals well, and there is no great advantage in $\stackrel{\mathcal{D}}{2}$ retaining the knee joint, especially in the very old. Recently I have carried out and been very impressed by the Stokes-Gritti amputation for $\stackrel{5}{9}$ gangrene in the aged. It is important in these $O$ people with a limited time to live that they should be up and about with the minimum delay. With an end-bearing Stokes-Gritti type of amputation and with a pylon artificial limb which can be made and fitted within two to three weeks of amputation, the patient will be getting around quite reasonably within a month, the longer femoral stump controlling the limb very much more satisfactorily than any 10 inch to 12 inch musclecovered femoral stump. A limb with flexion apparatus can be ordered and made but until this is delivered a home-made pylon enables the patient to go home and resume some of his or her activities at an early date. In the younger patient, $\forall$ a below-knee amputation should be aimed at and can usually be done with success. Apart from gangrene, persistent and demoralizing pain is a clear indication for amputation and this should not be delayed after a reasonable trial of conservative measures. It will be welcomed by the patient.

There are two types of diabetic gangrene Firstly where the diabetes happens to be presen in a patient with ischaemic gangrene, and secondl an infective variety where, as a result of diabetic neuritis, an infection of the foot has been allowed to progress. This condition is more of a cellulitis with infective gangrene and the blood supply is generally adequate. Treatment consists of a plantar incision and removal of affected digits and metatarsals and the provision of very adequate drainage together with chemotherapy. Healing occurs with remarkable rapidity. In the former variety the treatment differs in no way from the treatment of gangrene occurring in the nondiabetic.

It is important if possible to decide early whether amputation will be necessary in the near future and this depends largely on the patency or occlusion of the popliteal artery rather than on the tortuosity of the vessels at the wrist or the amount of calcification seen on X-ray. In fact, it is my $N$ impression that incipient or established gangrene $N$ occurring in a patient with a smooth, even radial artery is more serious and more frequently $\omega$ demands above-knee amputation than a similar condition in a patient with tortuous calcified vessels. Where gangrene has started or where $\$$ pain is severe and persistent in a patient with en absent popliteal pulse amputation should eb carried out above the knee without undue delay 
after preliminary treatment to improve the general health and to combat infection.

\section{BIBLIOGRAPHY}

HOMANS, J. (1939), 'Circulatory Diseases of the Extremities.' The Macmillan Co. New York.
LEARMONTH, J. R., BLACKWOOD, W., and RICHARDS, R. L. (1944), Edin. Med. Fourn., 51, I.

LEWIS, Thomas (1936), 'Vascular Disorders of the Limbs,' Macmillan and Co. London.

Medical Research Council Memorandum (1944), No. 13, 'Arterial Injuries.'

PRIESTLY, J. B. (1932), Fourn. Nerve and Mental Dis., 75, 137. RICHARDS, R. L. (1946), 'The Peripheral Circulation in Health and Disease,' $E$. and S. Livingstone (Edin.).

\title{
OBSERVATIONS ON THE ORGANIZATION OF CARDIOLOGY UNDER THE NATIONAL HEALTH SERVICE ACT
}

\author{
By Edmund Finsterbush, M.D.(Vienna), M.R.C.S.(Eng.), L.R.C.P.(London) \\ Consulting Cardiologist to the M:nistry of Pensions
}

It may be assumed that the organization of provincial hospitals will present ma y a difficult problem during the transitional stage of the inception of the National Health Service Act, 1946, owing to the great differences in organization, staffing, and clinical standards in municipal hospitals on the one hand and in voluntary hospitals on the other, as well as to the spirit of competition and isolation prevailing among institutions and doctors alike. Moreover, subjects such as psychiatry, dermatology, cardiology, etc. are novelties to a provincial hospital and will, as such, further aggravate the position. It may therefore not be out of place to outline some basic ideas of organization of a cardiological service.

\section{Pathology}

... for the signs of the dead body furnish invaluable data from which it is desirable sometimes to explain diagnosis, sometimes to justify, and always to learn.' (Sir George Newman, ' Notes on Medical Education' I9I8.)

There can be no real application of up-to-date clinical science without close and wholehearted co-operation of competent pathologists and radiologists with the physician. In the first place, post-mortem dissections must be performed as a routine, since they are undoubtedly the foremost tutor, highest examiner and corrector of the physician. Pathology is the sub-structure of diagnosis. (W. Boyd.) This may sound commonplace, yet must be constantly emphasized, for I am convinced that the lack of expert dissections in provincial hospitals is the main cause of the low clinical standard which prevails outside the teaching centres.
A few figures will clinch my statement. In a municipal borough somewhere in England in 1935 the total number of deaths was 1,368 ; of these 529 , (i.e. 38.6 per cent.) resulted from cardiovascular disease. In would-be compliance with the regulations made by the Registrar-General, the immediate cause of death in this group of 529 was certified as myocarditis in 25 , as syncope in 31 , and as cardiac dilatation in 21 cases. Arteriosclerosis as the sole cause of death occurred seven times. The extent of post-mortem studies in this group can be seen by the following. Out of the total of 529 deaths from cardiovascular disease 142 occurred in municipal hospitals, and $4 \mathrm{I}$ in the voluntary hospital. Among the former, two dissections took place, including one on the coroner's order; among the latter, seven dissections were performed spontaneously and one on the coroner's authority. In 1946 , the position in the same borough was that out of a total number of 1,526 deaths, 669 , (i.e. 43.8 per cent.), were ascribed to cardiovascular disease, among which 182 died in municipal hospitals, and 45 in the voluntary hospital. In the former, six post-mortem dissections were performed, including one on the coroner's order; in the latter twelve dissections were obtained on the hospital's own initiative, and six on the coroner's order. In this group of 669 deaths arteriosclerosis was certified as the immediate and sole cause 29 times ; myocarditis, both acute and chronic, was certified in 53 cases (sic), most ranging between 60 and 89 years of age. Some illustrative post-mortem diagnoses of 1946 may be quoted.

M.63 ' Cerebral haemorrhage due to toxaemia 